\title{
Improved concatenated (RS-CC) for OFDM systems
}

\author{
Mustafa Dh. Hassib ${ }^{1 \mathrm{a})}$, JS Mandeep ${ }^{1 \mathrm{~b})}$, Mardina Abdullah ${ }^{1 \mathrm{c})}$, \\ Mahamod Ismail $^{1 \mathrm{1})}$, Rosdiadee Nordin ${ }^{1 \mathrm{e})}$, and MT Islam ${ }^{2 \mathrm{f})}$ \\ ${ }^{1}$ Department of Electrical, Electronics, and Systems Engineering, Universiti \\ Kebangsaan Malaysia, 43600 Bangi, Selangor D.E., Malaysia \\ ${ }^{2}$ Institute of Space Science (ANGKASA), Universiti Kebangsaan Malaysia, Bangi \\ UKM, 43600, Selangor D.E., Malaysia \\ a)mdhassib@eng.ukm.my \\ b) mandeep@eng.ukm.my \\ c) mardina@eng.ukm.my \\ d) mahamod@eng.ukm.my \\ e) adee@eng.ukm.my \\ f) titareq@yahoo.com
}

\begin{abstract}
Orthogonal Frequency Division Multiplexing (OFDM) system became an important material for the current researchers. As like other communication systems, OFDM system needs to employ the channel coding to reduce the Bit Error Rate (BER) such as the concatenated Reed Solomon-Convolution code (RS-CC). To enhance more the BER problem, a Reed Solomon-Recursive Convolution code (RS-RCC) over Additive white Gaussian noise (AWGN) and fading channel has been used in this letter with same complexity of (RS-CC). The simulation results prove that the suggested method for different kinds of constellation mapping of various coding rates gives better BER performance than the old method represented by RS-CC.
\end{abstract}

Keywords: OFDM, concatenated (RS-CC), RCC and NCC

Classification: Wireless communication hardware

\section{References}

[1] D. N. K. Jayakody, L. O. Iheme, and E. A. Ince, "Coded QPSK-OFDM for Data Transmission over Fading Channels," 5th International Conference Information and Automation for Sustainability (ICIAFs), Colombo, Dec. 2010.

[2] "IEEE standard for local and metropolitan area networks Part 16: air interface for fixed broadband wireless access systems," IEEE Std. 802.16TM2009, May 2009.

[3] N. Nyirongo, W. Q. Malik, and D. J. Edwards, "Concatenated RSConvolutional Codes for Ultrawideband Multiband-OFDM," IEEE International Conf. Ultra-Wideband, Waltham, Sept. 2006.

[4] A. J. Viterbi, "Convolutional codes and their performance in communication systems," IEEE Trans. Commun. Technol., vol. COM-19, pp. 751772 , Oct. 1971.

[5] J. G. Proakis, Digital Communications, 4th ed., McGraw-Hill, Singapore, 2001. 
[6] H. Yoshikawa and Y. Kawadai, "Theoretical analysis of bit error probability for 4-state recursive systematic convolutional code with Max-Log MAP decoding," ISITA2010, Taichung, Taiwan, Oct. 2010.

\section{Introduction}

OFDM is a multicarrier transmission technique that has undergone development for more than five decades and is recognized as an excellent method for high-speed wireless data communication. There is also greater possibility that OFDM will be used in many application systems like IEEE $802.11 \mathrm{n}$ WLAN, UTRAN-LTE, and IEEE 802.16 mobile WiMax have received significant attention due to much higher spectral efficiency and transfer reliability [1]. OFDM system does not own low bit error rates (BER). Therefore, some form of forward error correction (FEC) must be used to lower the BER. This made bypass the input sequence through a (FEC) encoder for adding redundant bits in the transmitted message so that, if an instantaneous fades occurs in the channel, the data may still be recovered at the receiver by these redundant bits.

Due to the high complexity of low density purity check code (LDPC) encoder and convolution turbo code (CTC) decoder compare with low complexity of both encoder and decoder RS-CC. Therefore, the RS-CC has still particular interest in above system. Although, the (LDPC) and (CTC) among the known codes nearing the Shannon limit that can achieve very low BER for low SNR.

Over the years there are many applications and researches for RS-CC with OFDM like $[1,2,3]$ and all used Non Recursive Convolution code (NCC). Now, in this letter a recursive convolution code has been suggested instead of non recursive convolution code to improve the BER for OFDM system as it will be explained further in the following sections.

\section{Concatenated (RS-CC)}

The concatenated (RS-CC) scheme is a well-known type of error correction code (ECC) in which two (ECC) are used. The first one called the outer code (block codes), and the second one called the inner code (convolution codes) are concatenated to achieve comparatively high coding gain when comparing with single code. The benefits of using concatenation code are relatively to exploit the feature of block code (Reed-Solomon (RS)) and convolution codes (CC) to overcome the drawback of each other. In the transmitter, the total code rate for serial concatenation which is equal to the product of the two code rates, while in the receiver the Viterbi decoder used for the inner code and the RS decoder for the outer code. Furthermore, the serial concatenated decoders are used in the receiver because the serial concatenation scheme can share information between the concatenated decoders. 


\section{Proposed code}

The proposed code is based on the combine RS $(255,239,8)$ encoder using GF $\left(2^{8}\right)$ with RCC encoder shown in Fig. 1.a. The RCC obtained from the NCC encoder as shown in Fig. 1.b by feeding back one of its outputs to its input [2].

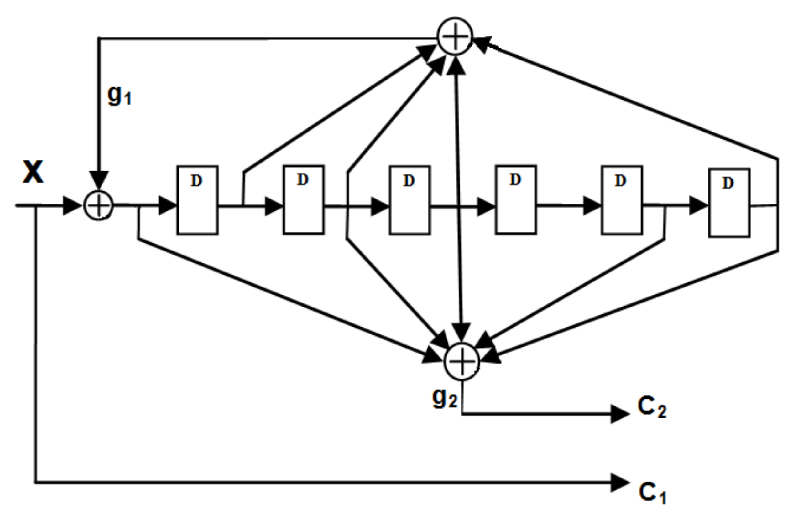

a. RCC Encoder.

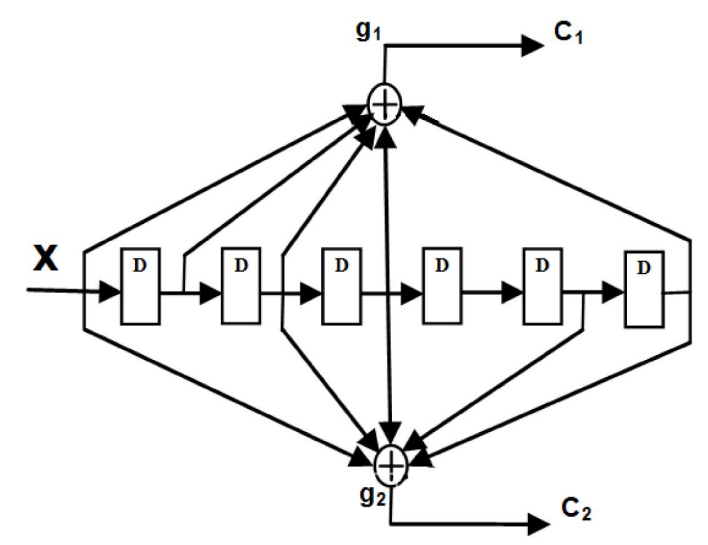

b. NCC Encoder.

Fig. 1. RCC and NCC Encoder.

It is clear from Fig. 1.a and Fig. 1.b the RCC and NCC encoders are represented by two generator sequences.

$$
\begin{aligned}
& g_{1}=[171]_{8}=1+D+D^{2}+D^{3}+D^{6} \\
& g_{2}=[133]_{8}=1+D^{2}+D^{3}+D^{5}+D^{6}
\end{aligned}
$$

Then the transfer function for NCC encoder for conventional code will be:

$$
G=\left[\begin{array}{ll}
g_{1} & g_{2}
\end{array}\right]=\left[1+D+D^{2}+D^{3}+D^{6} \quad 1+D^{2}+D^{3}+D^{5}+D^{6}\right]
$$

While the transfers function for RCC of this NCC encoder will be given by:

$$
G=\left[\begin{array}{ll}
1 & g_{2} / g_{1}
\end{array}\right]=\left[\begin{array}{ll}
1 & 1+D^{2}+D^{3}+D^{5}+D^{6} / 1+D+D^{2}+D^{3}+D^{6}
\end{array}\right]
$$

Where in the last equation for recursive convolution encoder, the first output represented by (g1) is feedback to the input, 1 denotes the systematic 
output and (g2) symbolizes the forward output. In the receiver, the Viterbi algorithm has been used to minimize the probability of word error for convolution code. It is a maximum likelihood decoding procedure based on finding the trellis path with the smallest distance between its digit sequence and the received sequence [4]. The union bound bit error probability $P_{b}$ at the output of the Viterbi decoder is given by [4].

$$
P_{b}<\sum_{d=d_{\text {free }}}^{\infty} \beta_{d} P_{2}(d)
$$

Where $\beta_{d}$ is a coefficient of transfer function $T(D, N)$ for the convolution code, $d_{\text {free }}$ denotes the free distance of the convention code, and $P_{2}(d)$ represented the pair-wise error probability. Where $p$ is the output bit error probability of QPSK demodulator [5].

$$
P_{2}(d)<[4 p(1-p)]^{d / 2}
$$

It can be seen from Eq. (5) that the output of the bit error probability for Viterbi decoder depends deeply on the encoder transfer function coefficient which is different from RCC to NCC. This difference leads to better bit error performance for RCC over the conventional convolution code due to the feedback of the RCC encoder to a primitive polynomial, because the primitive polynomial generates maximum length sequences, which add randomness to the code [6].

\section{Simulation and result}

In this section, we will apply the proposed code in section 3 as in IEEE 802.16 OFDM specification and it will be compared with the conventional concatenation code. The detailed structures of concatenated codes are displayed in Table I. There are six modes with different code rates and modulation schemes. The FFT/IFFT size is, 256, are divided into three groups 192 points for subcarriers, 56 points for guard and 8 for pilots. The Cyclic Prefix (CP) length is $1 / 4$ of OFDM symbol duration. The bandwidth is $5 \mathrm{MHz}$ and the sampling frequency is $8 \mathrm{MHz}$. The Puncturing, interleaver and the constellations of different modulations are implemented in the simulation. The bit pattern of puncturing was optimized to give better BER in high SNR when RCC was used. For example, in the second scheme the bit pattern for NCC are $\left[\begin{array}{llll}1 & 1 & 1 & 0\end{array}\right]$ has become $\left[\begin{array}{llll}1 & 0 & 1 & 1\end{array}\right]$ for RCC. The performance of all the six modes are evaluated under AWGN channel and fading channel (HIPERMAN, model C Large open space indoor and outdoor, NLOS conditions, and 150 ns delay spread).

The simulations present various BER vs. SNR plots, and it is divided to three plots for AWGN channel and one plot for fading channel according to the modulation level with different code rate to produce six modulation and coding schemes (MCS) as specified in Table I.

Fig. 2.a, b and c tell us the RS-CC concatenation code with RCC outperforms the RS-CC concatenation code with NCC for QPSK, 16 QAM and 


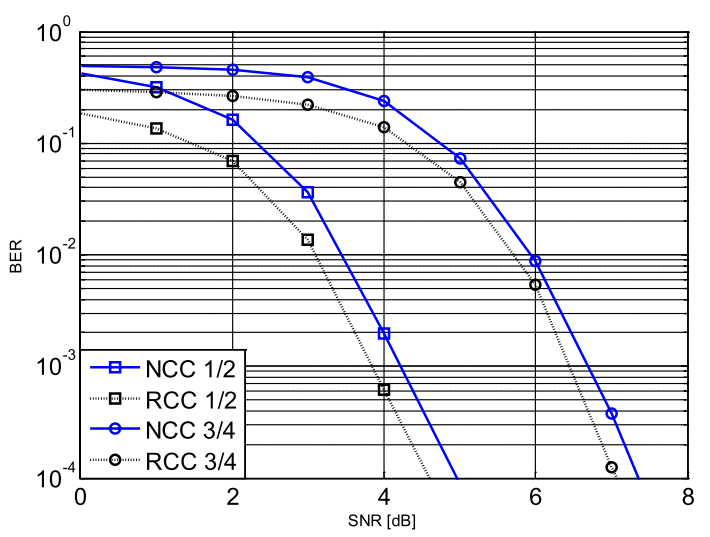

a. BER vs. SNR for QPSK in AWGN channel.

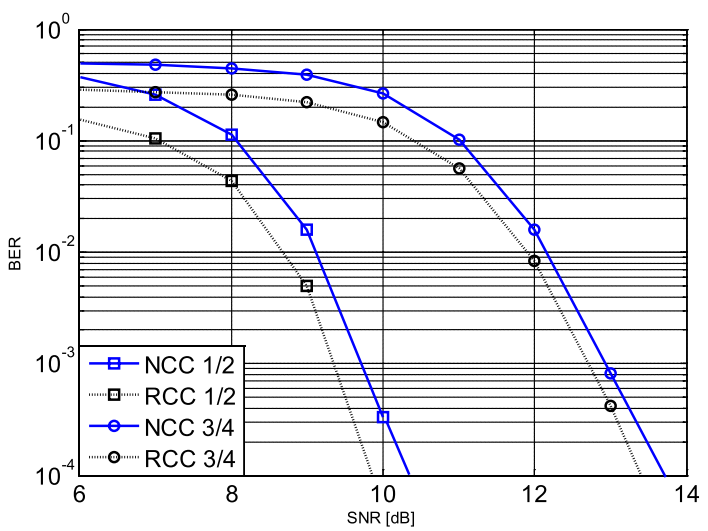

b. BER vs. SNR for 16QAM in AWGN channel.

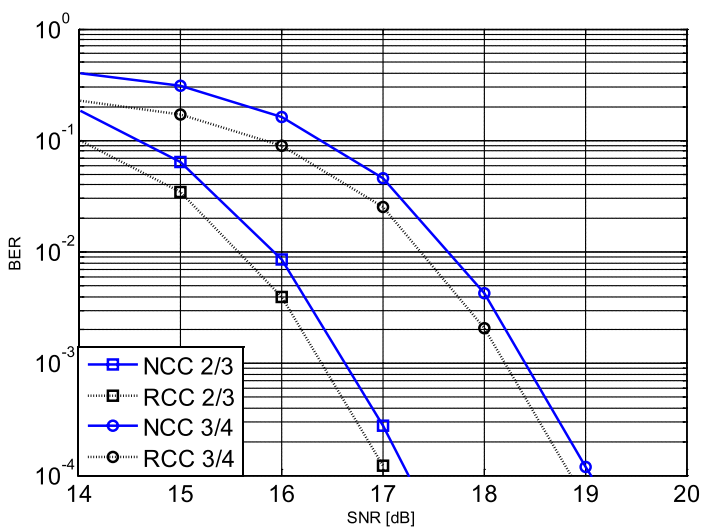

c. BER vs. SNR for 64QAM in AWGN channel.

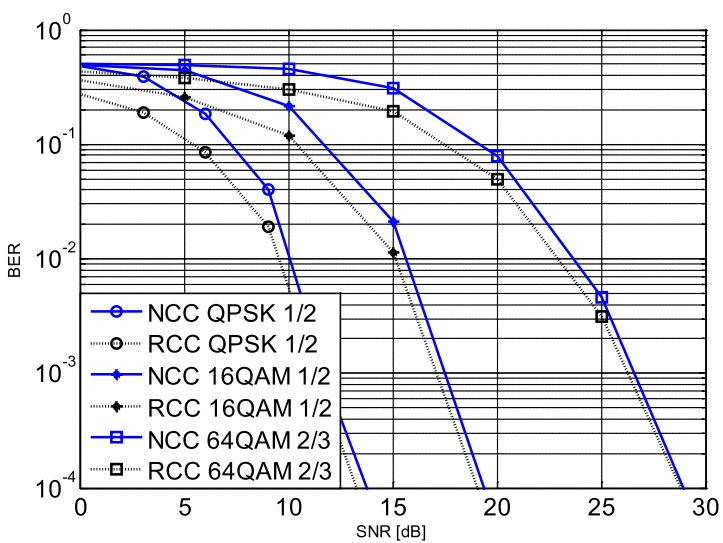

d. BER vs. SNR in Fading Channel for 1, 3 and 5 MCS as in table I.

(c) IEICE 2012

DOI: 10.1587/elex.9.538 Received February 01, 2012 Accepted February 25, 2012 Published March 25, 2012 
64 QAM modulations with different rates over AWGN channel. Fig. 2.a shows the gain enhancement when BER $10^{-3}$ for QPSK with rates $1 / 2$ and $3 / 4$ are $0.38 \mathrm{~dB}$ and $0.22 \mathrm{~dB}$ respectively. Moreover, from Fig. 2.b it can be seen that the gain enhancement equal $0.34 \mathrm{~dB}$ with rate $1 / 2$ and $0.25 \mathrm{~dB}$ with rate $3 / 4$ for 16 QAM when BER $10^{-3}$. Finally, we can see in Fig. 2.c that the gain enhancement when BER $10^{-3}$ for $64 \mathrm{QAM}$ with rate $2 / 3$ equal to $0.24 \mathrm{~dB}$ and with rate $3 / 4$ equal to $0.21 \mathrm{~dB}$.

Fig. 2.d show the simulation of proposed code over a fading channel for 1 , 3 and 5 MCS as in Table I. The simulation results show the code still powerful even when use in a fading channel. For example, the gain performances become $0.45 \mathrm{~dB}$ to QPSK of rate $1 / 2,0.34 \mathrm{~dB}$ to $16 \mathrm{QAM}$ of rate $1 / 2$ and $0.25 \mathrm{~dB}$ to $64 \mathrm{QAM}$ of rate $2 / 3$.

Table I. Different MCS for RS-CC concatenate code.

\begin{tabular}{|c|c|c|c|c|c|}
\hline MCS & Modulation & RS Code & $\begin{array}{c}\text { RS } \\
\text { code } \\
\text { Rate }\end{array}$ & $\begin{array}{c}\text { CC } \\
\text { Code } \\
\text { Rate }\end{array}$ & $\begin{array}{c}\text { Overall } \\
\text { Code } \\
\text { Rate }\end{array}$ \\
\hline 1 & QPSK & $(32,24,4)$ & $3 / 4$ & $2 / 3$ & $1 / 2$ \\
\hline 2 & QPSK & $(40,36,2)$ & $9 / 10$ & $5 / 6$ & $3 / 4$ \\
\hline 3 & $16-\mathrm{QAM}$ & $(64,48,8)$ & $3 / 4$ & $2 / 3$ & $1 / 2$ \\
\hline 4 & $16-\mathrm{QAM}$ & $(80,72,4)$ & $9 / 10$ & $5 / 6$ & $3 / 4$ \\
\hline 5 & 64-QAM & $(108,96,6)$ & $8 / 9$ & $3 / 4$ & $2 / 3$ \\
\hline 6 & 64-QAM & $(120,108,6)$ & $9 / 10$ & $5 / 6$ & $3 / 4$ \\
\hline
\end{tabular}

The gain enhancement was obtained due to the error pattern of RCC is more random than it of NCC so that the capability of RS code becomes more efficient for error correction. Also we can see the gain enmeshment depending on RS code capability to correct the errors, rates and code length of each mode scheme.

\section{Conclusion}

In this letter, we used RCC to improve the performance of RS-CC concatenated codes defined in IEEE 802.16 OFDM specification on different types of constellation mappings with various coding rates over AWGN and fading channel The comparative analysis of the BER shows that the gain enhancement has been improved by using new coding method for different rate of modulation techniques from $0.21 \mathrm{~dB}$ to $0.38 \mathrm{~dB}$ in AWGN channel and from $0.25 \mathrm{~dB}$ to $0.45 \mathrm{~dB}$ over fading channel. Also the power consumption of the system was reduced with no add complexity for the system. 